\title{
The differential diagnosis of primary electrical diseases from seizures in childhood
}

Keywords: Congenital long QT syndrome; Brugada syndrome; catecholaminergic polymorphic ventricular tachycardia; sudden cardiac death; epilepsy

Received: 28 June 2009; Accepted: 9 August 2009; First published online 13 January 2010

\section{Dear Sir,}

The recent diagnosis of a catecholaminergic polymorphic ventricular tachycardia in a young boy, considered for several years as epileptic, and whose sister died suddenly after a long history of refractory anti-epileptic, has encouraged us to write this letter. Cardiac channelopathies appear to be often underdiagnosed, especially during childhood. We focus here on their relevance to seizures in children, and the importance of considering and investigating them as a differential diagnosis from epilepsy.

Clinical data supports the notion that those suffering from epilepsy have a two- to three-fold increase of premature death than those without epilepsy. The most common mode of sudden and unexpected death, in fact, is epilepsy. The pathophysiology of this happening in epileptics remains obscure, but cardiac arrhythmias are likely to play a role during seizures. ${ }^{1}$

Cardiac channelopathies, principally represented by the congenital long QT syndrome, Brugada syndrome, and catecholaminergic polymorphic ventricular tachycardia, are a group of inherited primary electrical diseases predisposing to sudden cardiac death by ventricular arrhythmias occuring in structurally normal hearts. ${ }^{2}$ They are sometimes misdiagnosed as epilepsy, especially during childhood, as arrhythmia-related syncope can lead to convulsions.

Evidence supporting the possible misdiagnosis of long QT syndrome as epilepsy is now well documented. $^{3-6}$ Brugada syndrome and catechol-

Correspondence to: Alban-Elouen Baruteau, MD, Département de Cardiologie et Maladies Vasculaires, Centre Cardio-pneumologique, Hôpital Pontchaillou, Centre Hospitalier Universitaire de Rennes, 2 rue Henri Le Guilloux, 35033 Rennes cedex, France. Tel: (+33)299282525; Fax: (+33)299282510; E-mail: alban.baruteau@wanadoo.fr aminergic polymorphic ventricular tachycardia can also present as seizures in childhood. Primary electrical diseases, however, remain underdiagnosed. When properly diagnosed, anti-arrhythmic treatment can efficiently prevent sudden cardiac death, while familial screening can lead to early diagnosis of asymptomatic relatives. That is why investigations of seizures in a child should include a 12-lead electrocardiogram at rest, and also during exercise should the seizures occur in an adrenergic context, such as exercise, emotion, or stress.

Brugada syndrome is characterized by a elevation of the ST segments in the right precordial leads, and carries a predisposition to sudden cardiac death, particularly in children and young adults. Its affects from 1 to 5 per 10,000 inhabitants worldwide. ${ }^{7}$ Ventricular arrhythmias typically occur at rest, especially while sleeping. ${ }^{7}$ Fever represents the most important precipitating factor for arrhythmic events in children, and life-threatening arrhythmias can masquerade as febrile seizures. ${ }^{8,9}$ It has been diagnosed in patients with a history of drug-resistant seizures. $^{10,11}$

Catecholaminergic polymorphic ventricular tachycardia is characterized by stress-induced ventricular tachyarrhythmias, and usually occurs during childhood. If untreated, the estimated mortality is from one-third to one-half of those afflicted before the age of 30 years. ${ }^{12,13}$ The resting electrocardiogram is normal. Diagnosis is through the exercise electrocardiogram, which shows a typical bidirectionnal ventricular tachycardia. ${ }^{12,14}$ Family history is positive in three-quarters of cases, and almost half of the afflicted children are initially considered to be epileptic, and are often treated as such for several times without control of attacks. ${ }^{15}$

Long QT syndrome, Brugada syndrome and catecholaminergic polymorphic ventricular tachycardia 
should all be investigated, therefore, as part of the differential diagnosis of paediatric seizures. A family history of seizures is not indicative of epilepsy, as it can be due to misdiagnosed inherited arrythmias. We submit, therefore, that a cardiological assessment of presumed epileptic children should always be considered, especially in case of drug-refractory seizures, and in case of a family history of seizures or sudden death.

Alban-Elouen Baruteau ${ }^{1,2,3,4}$, Julien Baruteau ${ }^{5,6}$

${ }^{1}$ CHU Rennes, Service de Cardiologie et Maladies Vasculaires, Rennes, F-35000, France

${ }^{2}$ Université de Rennes 1, LTSI, Rennes, F-35000, France

${ }^{3}$ INSERM, CIC-IT 804, Rennes, F-35000, France

${ }^{4}$ INSERM, U915, Nantes, F-44000, France

${ }^{5}$ Assistance Publique - Hôpitaux de Paris, Hôpital

Robert Debré, Service de Neurologie Pédiatrique et des Maladies Héréditaires du Métabolisme, Paris, F-75000,

France

${ }^{6} \mathrm{CHU}$ Toulouse, Service d'Hépato-Gastro-Entérologie Pédiatrique et des Maladies Héréditaires du Métabolisme, Toulouse, F-31000, France ${ }^{7}$ INSERM, U642, Rennes, F-35000, France

\section{References}

1. Stollberger C, Finsterer J. Cardiorespiratory findings in sudden unexplained/unexpected death in epilepsy (SUDEP). Epilepsy Res 2004; 59: 51-60.

2. Lehnart SE, Ackerman MJ, Benson DW $\mathrm{Jr}$, et al. Inherited arrhythmias: a National Heart, Lung, and Blood Institute and Office of Rare Diseases workshop consensus report about the diagnosis, phenotyping, molecular mechanisms, and therapeutic approaches for primary cardiomyopathies of gene mutations affecting ion channel function. Circulation 2007; 116: $2325-2345$.

3. Ghosh A, Agarwala BN. Torsades de Pointes in a child. Pediatr Cardiol 2009; 30: 553-554.

4. Johnson JN, Hofman N, Haglund CM, Cascino GD, Wilde AA, Ackerman MJ. Identification of a possible pathogenic link between congenital long QT syndrome and epilepsy. Neurology 2009; 72: 224-231.

5. Levine E, Rosero SZ, Budzikowski AS, Moss AJ, Zareba W, Daubert JP. Congenital long QT syndrome: considerations for primary care physicians. Cleve Clin J Med 2008; 75: 591-600.

6. Maccormick JM, McAlister H, Crawford J, et al. Misdiagnosis of long QT syndrome as epilepsy at first presentation. Ann Emerg Med 2009, Mar 10 [Epub ahead of print].

7. Antzelevitch C, Brugada P, Borggrefe M, et al. Brugada syndrome: report of the second consensus conference: endorsed by the Heart Rhythm Society and the European Heart Rhythm Association. Circulation 2005; 111: 659-670.

8. Probst V, Denjoy I, Meregalli PG, et al. Clinical aspects and prognosis of Brugada syndrome in children. Circulation 2007; 115: 2042-2048.

9. Skinner JR, Chung SK, Nel CA, et al. Brugada syndrome masquerading as febrile seizures. Pediatrics 2007; 119: e1206-e1211.

10. Fauchier L, Babuty D, Cosnay P. Epilepsy, Brugada syndrome and the risk of sudden unexpected death. J Neurol 2000; 247: 643-644.

11. Girard S, Cadena A, Acharya Y. Woman with Brugada syndrome and epilepsy: a unifying diagnosis? South Med J 2008; 101: $1150-1153$.

12. Francis J, Sankar V, Nair VK, Priori SG. Catecholaminergic polymorphic ventricular tachycardia. Heart Rhythm 2005; 2: 550-554.

13. Leenhardt A, Lucet V, Denjoy I, Grau F, Ngoc DD, Coumel P. Catecholaminergic polymorphic ventricular tachycardia in children. A 7-year follow-up of 21 patients. Circulation 1995; 91: $1512-1519$.

14. Liu N, Ruan Y, Priori SG. Catecholaminergic polymorphic ventricular tachycardia. Prog Cardiovasc Dis 2008; 51: 23-30.

15. Celiker A, Erdogan I, Karagoz T, Ozer S. Clinical experiences of patients with catecholaminergic polymorphic ventricular tachycardia. Cardiol Young 2009; 19: 45-52. 